\title{
A THEORETICAL CONNECTION BETWEEN THE NOISY LEAKY INTEGRATE-AND-FIRE AND THE ESCAPE RATE MODELS: THE NON-AUTONOMOUS CASE
}

\author{
Grégory Dumont ${ }^{1}$, JACQues Henry ${ }^{2}$ \\ AND CARMEN OANA TARNICERIU ${ }^{3,4, *}$
}

\begin{abstract}
Finding a mathematical model that incorporates various stochastic aspects of neural dynamics has proven to be a continuous challenge. Among the different approaches, the noisy leaky integrate-and-fire and the escape rate models are probably the most popular. These two models are generally thought to express different noise action over the neural cell. In this paper we investigate the link between the two formalisms in the case of a neuron subject to a time dependent input. To this aim, we introduce a new general stochastic framework. As we shall prove, our general framework entails the two already existing ones. Our results have theoretical implications since they offer a general view upon the two stochastic processes mostly used in neuroscience, upon the way they can be linked, and explain their observed statistical similarity.
\end{abstract}

Mathematics Subject Classification. 35Q84, 35Q92.

Received July 8, 2019. Accepted April 27, 2020.

\section{INTRODUCTION}

Stochasticity is nowadays considered as an unavoidable ingredient that cannot safely be ignored in the study of biological systems [36]. Conceptual frameworks are thus needed to appropriately render out the presence of random fluctuations. In mathematical neuroscience, the noisy behavior of neuronal dynamics [23] makes the use of stochastic processes necessary. The problem is then to identify the key elements one needs to incorporate in models in order to reproduce the experimentally observed emergent patterns. A great deal of attention has been devoted to the precise analytical expression of these fluctuations. A generally accepted formalism to express the variability present in neural dynamics has not been found yet, but among the most notable models dealing with this aspect stand out the noisy leaky integrate-and-fire (NLIF) [8,9], and the escape rate models [25].

Keywords and phrases: Neural noise, noisy leaky integrate-and-fire model, escape rate model.

1 École Normale Supérieure, Group for Neural Theory, 45 Rue d'Ulm, 75005 Paris, France.

2 INRIA team Carmen, INRIA Bordeaux Sud-Ouest, 200 Avenue de la Vieille Tour, 33405 Talence cedex, France.

3 Department of Mathematics and Informatics, "Gheorghe Asachi" University of Iaşi, Bulevardul Carol I 11, 700506 Iaşi, Romania.

${ }^{4}$ Interdisciplinary Research Department, Field - Sciences, "Alexandru Ioan Cuza" University of Iaşi, Lascăr Catargi 54, Iaşi, Romania.

* Corresponding author: tarniceriuoana@yahoo.co.uk 
The modelling assumptions on which these two models were built correspond to different mechanisms of noise action over the neural cell. As it is well known, there are various sources of noise that influence the neural dynamics [29], but a simplistic categorization of them in concordance with the localization (external or internal) of the origin of the randomness has been popularized [26]. In this classification, external noise stands for the sources of randomness that are mainly due to the fluctuation of synaptic input over a single unit, internal noise however, usually refers to the intrinsic stochastic mechanisms that allow the cell to initiate an action potential. Accordingly, the NLIF model renders out the effect of an external noise while the escape rate model is used to express the internal neural noise. Of course, the use of each of these models comes with its own advantages.

The NLIF model - a model going back to Lapique's work in $1907[1,3,7]$ - describes neurons as simple electrical circuits consisting in a capacitor in parallel with a resistor driven by a noisy input. Written in the language of stochastic differential equations, it gives rise to a Langevin equation [28] completed with a reset mechanism that mimics the onset of an action potential. Related to a stochastic variable satisfying the Langevin equation, stands the probability density function that expresses the likelihood of the state variable to take on a specific value [24]. For the NLIF model, its associated pdf follows the well-known Fokker-Planck (FP) equation [24].

The escape rate model - a formalism proposed by W. Gerstner and J.L. van Hemmen in 1992 [25] - introduces a rather different approach, in which the neuron's dynamics remain deterministic but the generation of an action potential is of stochastic nature. The firing probability is then described by a stochastic intensity of firing or hazard function which depends on the momentary distance between the membrane potential and a formal firing threshold $[25,26]$. In this setting, the neuron fires in a stochastic manner [26]. The pdf associated to the escape rate model satisfies a pure transport equation along the deterministic trajectories, and a term to express the probabilistic nature of spike initiation is added. This equation, completed with a nonlocal boundary condition that models the reset mechanism, leads to an age structured (AS) system, where the age variable accounts for the time elapsed since the neuron had its last spike [25].

At a first sight, the two stochastic processes reminded above seem essentially different, both in form and in modelling assumptions. Despite their contrasting nature, it has been observed that they can behave in a surprisingly comparable fashion [34]. Which generates the main question that we address in the present paper: is there an underlying reason for the reported similar statistical activity of the two models?

In our previous work $[19,20]$, we made a first step in this direction. We have highlighted an unforeseen relationship between the two modelling frameworks in the case of a constant stimulus acting over the neural cell, but we have left several open questions, especially in the context of time dependent stimuli, which will be the case analysed in the present paper. We introduced there integral transforms between the solutions of Fokker-Planck and Age-Structured systems using kernels defined by the first passage time problem. Considering a time-independent stimulus, we could build a transformation valid at any time. However, when considering a time-dependent stimulus instead, these time transformations are not possible any more, and, as we pointed out in [19], time dependence of the kernels becomes necessary. For this reason, in the current paper, we have introduced a two-dimensional stochastic processes and used the corresponding Kolomogorov equations (forward and backward in time) to construct new integral transforms. As a consequence, we now have a more general transform to the one previously introduced in [20].

The paper is structured as follows: We shall first remind the NLIF and the escape rate models as they are usually encountered in the literature. Next, we introduce a two dimensional stochastic description of the neural state, and, starting from there, we show that our general stochastic framework entails the two already existing ones. Integral transforms, similar to those treated in $[19,20]$, that set the connections between the two formalisms (FP equation and AS systems) are proven for the general time-dependent system. In addition to our previous results, we make use here of the dual problem to the first passage time problem in the non-autonomous case. Numerical simulations have been performed for each model to illustrate the corresponding dynamics. We finish with a discussion on how the proposed transform can help to estimate the level of the stimulus over a neuronal population when only observing the number of neurons firing at an instant $t$ and have been silent since then. 


\section{TWO STANDARD MODELS FOR NEURAL NOISE}

\subsection{Fokker-Planck equation}

The first process that we shall refer to the NLIF model is given by a Langevin equation which describes the dynamics of the neural membrane in the subthreshold regime, subject to a time dependent stimulus:

$$
\frac{\mathrm{d}}{\mathrm{d} t} v(t)=(\mu(t)-v)+\sigma \xi(t)
$$

where $\mu(t)$ is the mean of the external stimuli, which will be considered bounded in the rest of the paper, $\sigma$ is the noise intensity of the external stimuli, and $\xi$ is a normalized Gaussian white noise

$$
\langle\xi(t)\rangle=0, \quad\left\langle\xi(t) \xi\left(t^{\prime}\right)\right\rangle=\delta\left(t-t^{\prime}\right)
$$

Here, the brackets denote a time-dependent ensemble averaging over possible realizations of the stochastic process.

It is supposed that, whenever the potential variable reaches a fix threshold value, in this paper normalized to 1 , the neuron emits a spike and the potential is immediately reset to another given value $v_{r}<1$. The firing mechanism of the neuron is therefore expressed by a discontinuous reset process:

$$
\text { if } \quad v \geq 1 \text { then } v \mapsto v_{r} .
$$

After spiking, the process continues following the same dynamics (2.1) up until the reset condition (2.2) is again fulfilled. Thus, the dynamics of the process are influenced on each inter spike interval by the last firing time and by the time course of the stimulus $\mu(t)$ at any instant of the subinterval. This fact situate us in the case of input dependent renewal processes [26].

By associating to the stochastic variable $v$ a pdf $p(t, v)$, it is found that the pdf satisfies the FP equation [6]:

$$
\frac{\partial}{\partial t} p(t, v)+\frac{\partial}{\partial v}[(\mu(t)-v) p(t, v)]-\frac{\sigma^{2}}{2} \frac{\partial^{2}}{\partial v^{2}} p(t, v)=\delta\left(v-v_{r}\right) r(t)
$$

where an absorbing boundary condition at the spiking threshold is imposed in order to express the immediate reset of the potential variable,

$$
p(t, 1)=0,
$$

whereas the flux at this boundary defines the firing rate:

$$
r(t)=-\frac{\sigma^{2}}{2} \frac{\partial}{\partial v} p(t, 1)
$$

At the lower boundary, the no-flux condition is imposed:

$$
\lim _{v \rightarrow-\infty}\left[(v-\mu(t)) p(t, v)+\frac{\sigma^{2}}{2} \frac{\partial}{\partial v} p(t, v)\right]=0 .
$$

An initial repartition is assumed as known:

$$
p(0, v)=p_{0}(v)
$$



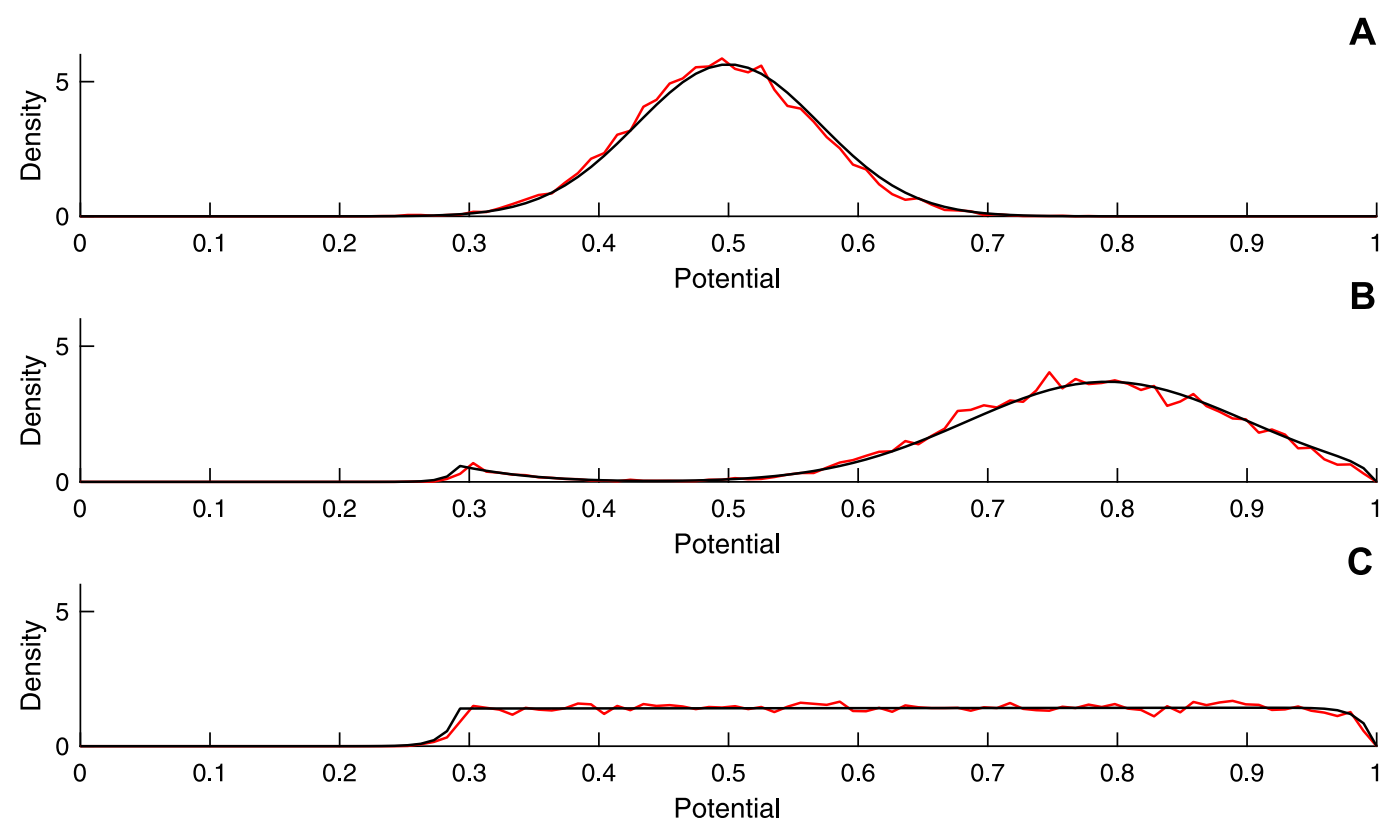

Figure 1. Simulations of the NLIF model (2.1), (2.2) and of its associated pdf that satisfies the FP model (2.3)-(2.6) in the case of constant in time stimulus. Comparison between the density function extracted from several realizations of the stochastic process $(2.1),(2.2)$ in red, and a simulation of the density given by its associated FP equation (2.3)-(2.6) in black. A Gaussian was taken as initial condition; the parameters of the simulations are: $v_{r}=0.3, \mu=3, \sigma=0.15$, the different plots A-B-C correspond to different time instants: $t=0, t=0.05, t=0.3$.

for which the normalization condition is imposed, due to the interpretation of $p$ as a pdf:

$$
\int_{-\infty}^{1} p_{0}(v) \mathrm{d} v=1
$$

It can be easily shown that, once the condition (2.7) is assumed, then the conservation property of the solution to the system (2.3)-(2.6) takes place at any time:

$$
\int_{-\infty}^{1} p(t, v) \mathrm{d} v=1
$$

We present in Figure 1 a simulation of the FP equation (2.3)-(2.6), in black, compared with Monte Carlo simulations for the stochastic NLIF model (2.1), (2.2), in red, for the time independent stimulus case. A Gaussian was taken as initial condition (see Fig. 1A). Under the drift and the diffusion effects, the density function gives a non zero flux at the threshold that is reset according to the condition (2.2). This effect can be seen clearly in the second panel of the simulation presented in Figure 1B. Asymptotically, the solution reaches a stationary repartition (see Fig. 1C).

In Figure 2, a simulation of the NLIF model (2.1), (2.2) in the case of constant in time stimulus is presented. The first panel (Fig. 2A) illustrates the dynamics of the stochastic process under different noise realizations. As expected, the corresponding dynamics are different and the neuron spikes at different times. In the second panel (Fig. 2B), the spiking activity of the same cell is extracted from a bigger number of trials. Finally, in the 

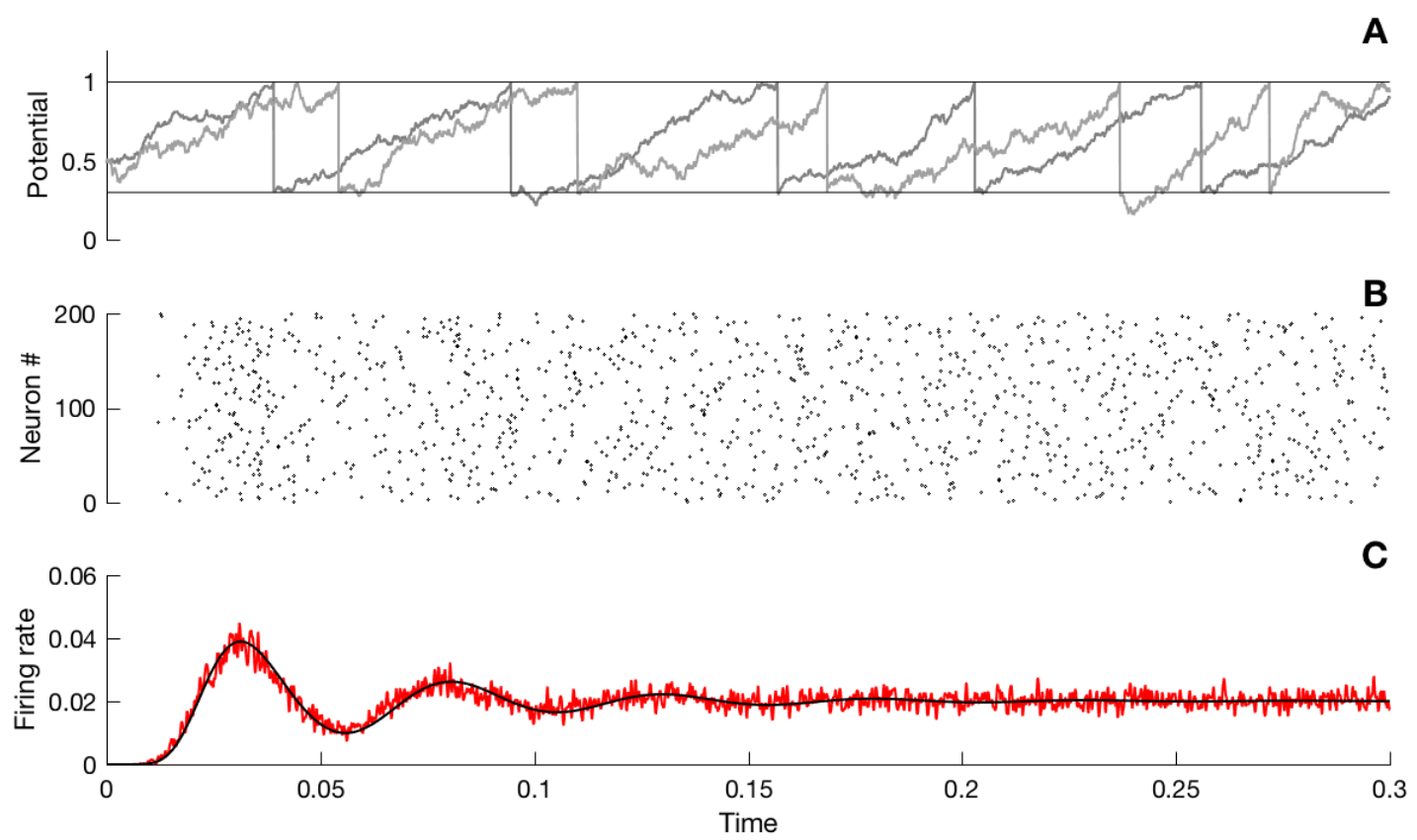

FiguRE 2. Simulations of the NLIF model (2.1), (2.2) and of its associated pdf that satisfies the FP model (2.3)-(2.6) in the case of constant in time stimulus. (A) Illustration of the noise action onto the membrane potential via two realizations of the stochastic process $(2.1),(2.2)$. (B) Spiking activity generated via several realizations of the stochastic process (2.1), (2.2), each dot representing the realization of an action potential. (C) Comparison between the activity extracted from 200 realizations of the stochastic process $(2.1),(2.2)$ in red, and the activity given by the FP equation (2.3)-(2.6) in black. A Gaussian was taken as initial condition; the parameters of the simulations are: $v_{r}=0.3, \mu=3, \sigma=0.15$.

last panel (Fig. 2C), the corresponding spiking activity is compared with the firing rate obtained from the FP model (2.3)-(2.6).

In Figure 3, a comparison between the spiking activities generated by the NLIF model and by the FP equation subject to a time-dependent stimulus is presented. The first panel (Fig. 3A) shows the time evolution of the stimulus. In the second panel (Fig. 3B), the spiking activity of the cell is displayed, from which we can extract the firing rate. A comparison with the spiking activity generated by the FP equation is shown in the last panel (Fig. 3C).

\subsection{Age-structure formalism}

Contrary to the case of stochastic trajectories completed with fix firing threshold, which was the case illustrated in the previous subsection, the escape rate models consider deterministic trajectories combined with a probabilistic firing mechanism [25]. Without restraining the generality, we can consider the state variable for the deterministic trajectories as the time elapsed since the last spike, that we will generically call age:

$$
\frac{\mathrm{d}}{\mathrm{d} t} a(t)=1
$$

The probability that an action potential occurs during a small time interval is computed via the hazard function $S(t, a)$ that gives the probability of spiking of a neuron that has at time t the age $a$. More precisely, during the 

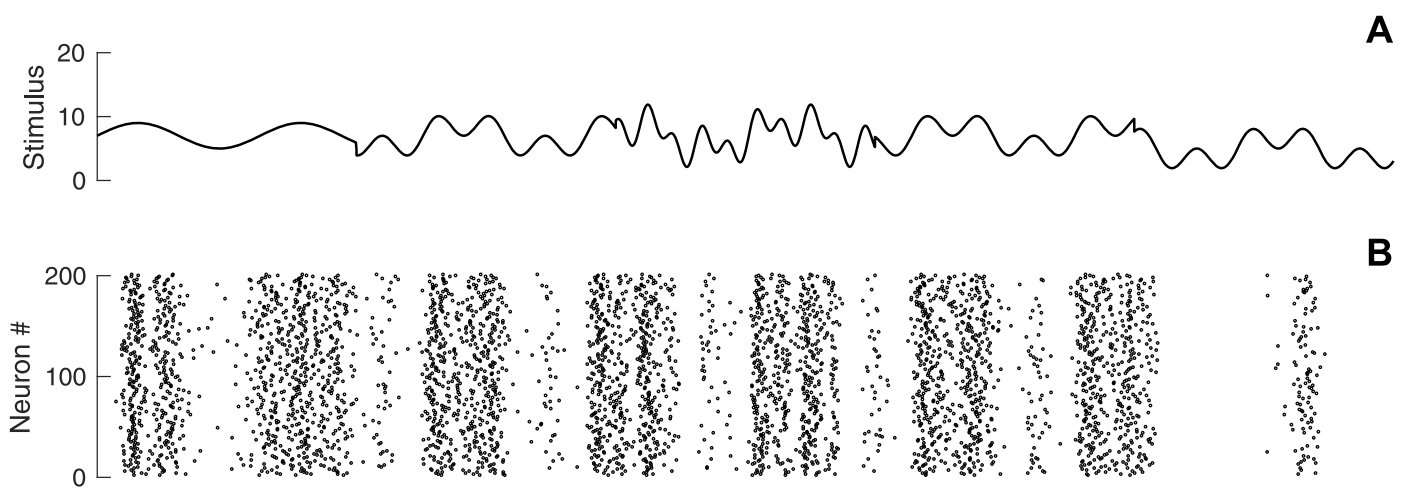

B

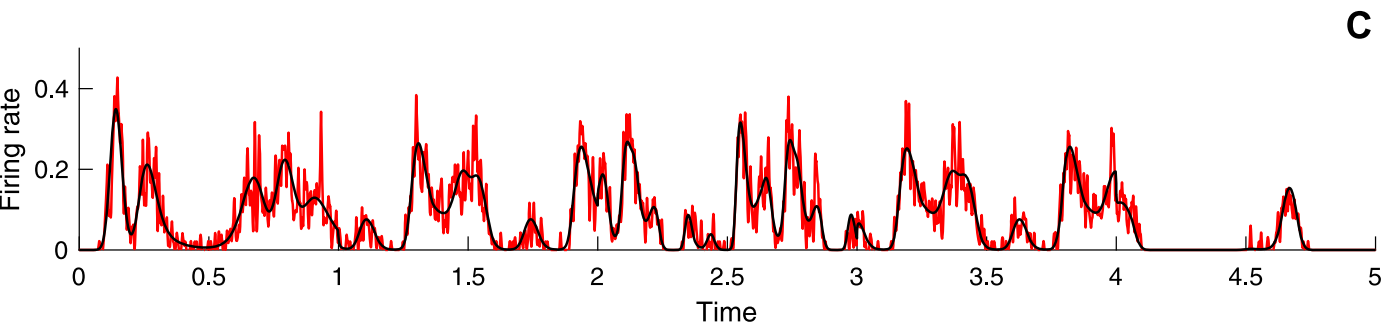

Figure 3. Comparison between the neural activity generated via 200 realizations of the stochastic process (2.1), (2.2) and the one generated by the FP model (2.3)-(2.6) for a time dependent stimulus $\mu(t)$. (A) Time evolution of the stimulus. (B) Activity obtained from 200 realizations of the stochastic process (2.1), (2.2). (C) Comparison between the firing rate obtained from the stochastic process (2.1), (2.2) in red, and the one given by the FP model (2.3)-(2.6) in black. A Gaussian was taken as initial condition; the parameters of the simulations are: $v_{r}=0.5$, $\sigma=0.2$.

time interval $(t, t+\mathrm{d} t)$, a spike occurs with probability $S(t, a(t)) \mathrm{d} t$, and, consequently, the age is reset to zero immediately after:

If the spike occurs at time $t$ then $a \mapsto 0$.

Note that, as in the NLIF case, the process described here classifies as an input dependent renewal process, due to the time dependence of the hazard rate. In the case of a constant stimulus acting over the neural cell, the dynamics of the process will only be influenced by the last firing time, therefore, only by the age of the system. It is not the same case when a time dependent input is taken, since in that case, the dynamics are also time dependent due to the time course of the stimulus.

The associated probability density function, $n(t, a)$, satisfies the following PDE $[25,26]$ :

$$
\frac{\partial}{\partial t} n(t, a)+\frac{\partial}{\partial a} n(t, a)+S(t, a) n(t, a)=0,
$$

where the first two terms describe the pure transport along the deterministic trajectories (2.9), and the last term accounts for the probability of spiking.

A non-local boundary condition models the reset mechanism at age zero after the neuron spikes:

$$
n(t, 0)=r(t)=\int_{0}^{\infty} S(t, a) n(t, a) \mathrm{d} a,
$$



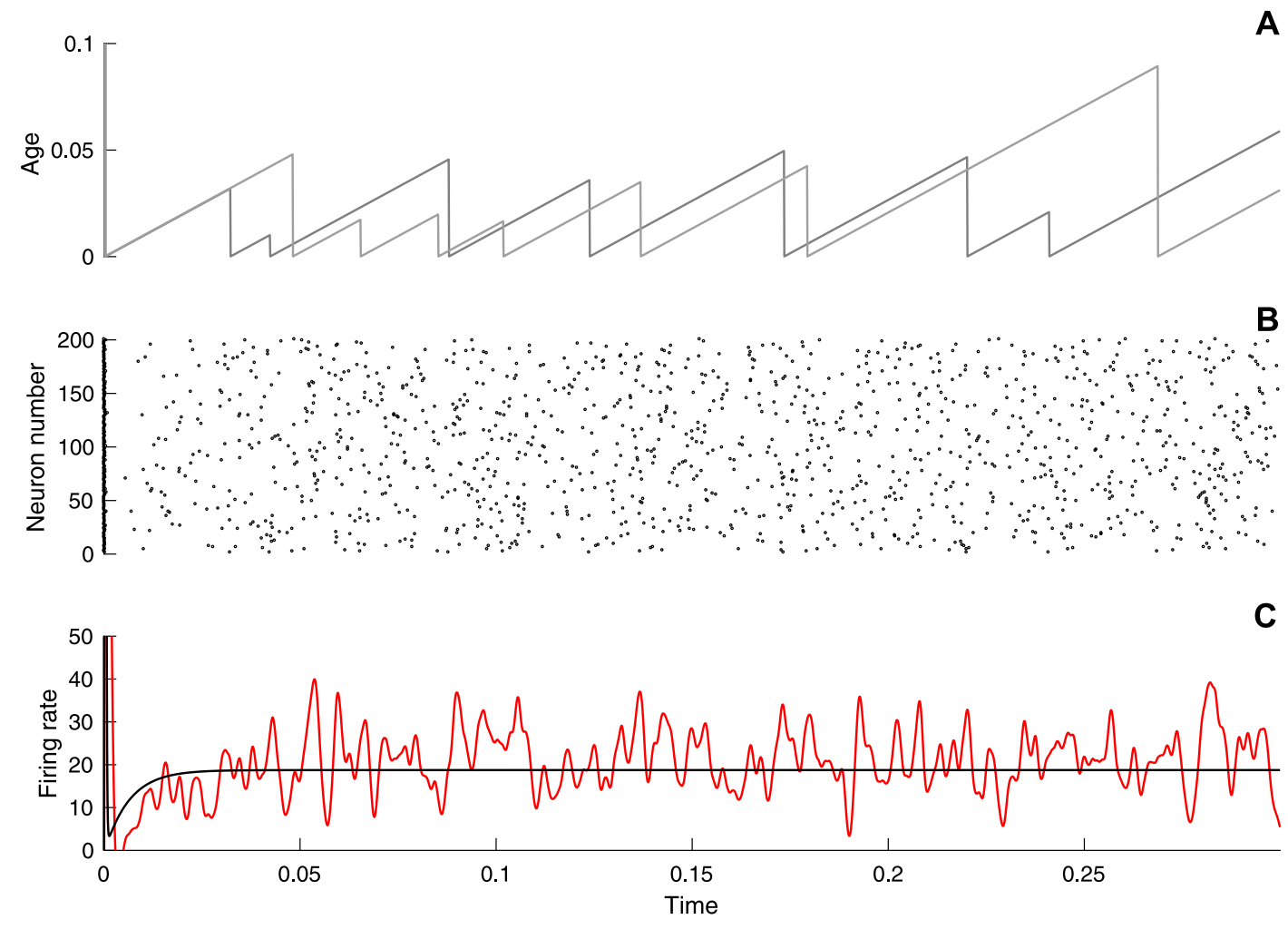

Figure 4. Simulations of the stochastic process (2.9), (2.10) and of its associated AS system (2.11)-(2.13) in the case of constant stimulus. (A) Illustration of the noise action onto the age dynamics (2.9), (2.10). (B) Spiking activity generated via several realizations of the stochastic process $(2.9),(2.10)$. (C) Comparison between the activity extracted from several realizations of the stochastic process (2.9), (2.10) in red, and a simulation of the firing rate given by its associated AS system (2.11)-(2.13) in black. A Gaussian was taken as initial condition; the functions and the parameters of the simulation are: $S(a)=\exp (h-V(a))$, with $V(a)=$ $-\log (1-\exp (-a / \tau))$ and $\tau=30, h=10$.

where the right hand side of the above equation defines the firing rate of the system.

As before, an initial repartition is taken as known:

$$
n(0, a)=n_{0}(a)
$$

The conservation property of the system (2.11)-(2.13) takes place as well. Namely,

$$
\int_{0}^{\infty} n(t, a) \mathrm{d} a=1
$$

at any $t$, as soon as

$$
\int_{0}^{\infty} n_{0}(a) \mathrm{d} a=1
$$



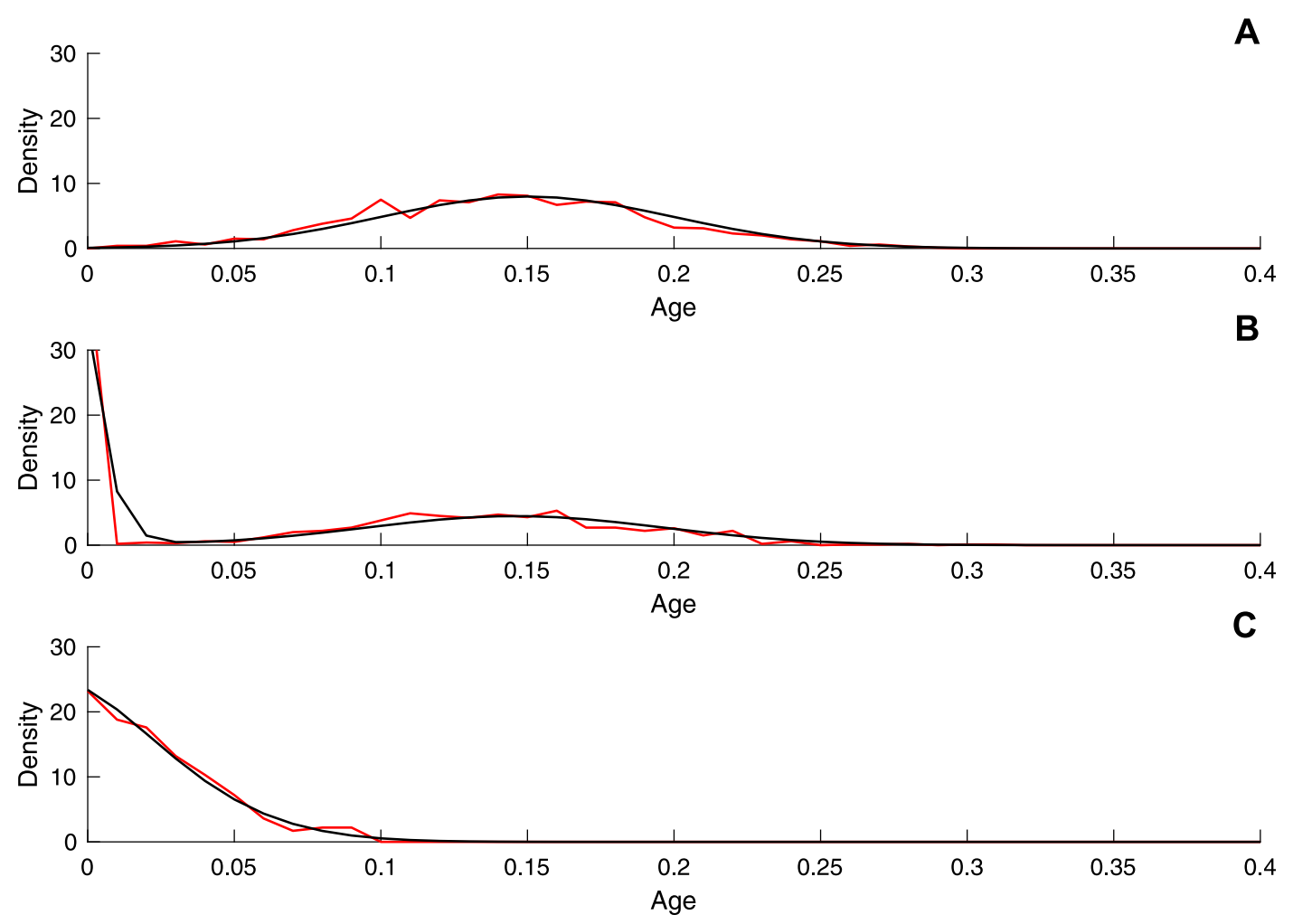

FIgURE 5. Simulations of the stochastic process (2.9), (2.10) and of its associated AS system (2.11)-(2.13) in the case of constant stimulus. Comparison between the density function extracted from several realizations of the stochastic process (2.9), (2.10) in red, and a simulation of the density function given by its associated AS system (2.11)-(2.13) in black. A Gaussian was taken as initial condition; the functions and the parameters of the simulation are: $S(a)=\exp (h-V(a))$, with $V(a)=-\log (1-\exp (-a / \tau)), \tau=30$ and $h=10$. The different plots, A-B-C, correspond to different time instants: $t=0, t=0.005, t=0.1$.

We present in Figure 4 a numerical simulation of the model (2.11)-(2.13) in the particular case when the hazard rate depends only on age, i.e. $S(t, a) \equiv S(a)$. Again, we have made a comparison between the firing rates given by the stochastic process (2.9), (2.10) (red curve) and by the corresponding system (2.11)-(2.13) (black curve). The first panel (Fig. 4A) presents two simulations of the evolution in time of the age variable for the same neuron. As expected, due to the probabilistic firing process, the corresponding dynamics have different spike times. In the second panel (Fig. 4B), the spiking activity of the same cell is extracted from a large number of trials. In the last panel (Fig. 4C), the corresponding spiking activity is compared with the firing rate given by the AS system (2.12).

In Figure 5 a simulation of the problem (2.11)-(2.13), in black, compared with one of the stochastic process (2.9), (2.10), in red, is presented. The simulation starts with a Gaussian as initial condition (Fig. 5A). Due to the spiking process, the age of the neuron is reset to zero, which is well perceived in Figure 5B. As expected from the model, the density function converges to an equilibrium repartition (Fig. 5C).

In Figure 6, a numerical simulation of the stochastic process defined by (2.9), (2.10), in the case of a time dependent hazard rate is illustrated. Note that the neuron never fires exactly at the same age, since its probability to fire (escape) is purely stochastic. The first panel (Fig. 6A) shows the time evolution of the stimulus, which was taken as a sum of Heaviside functions multiplied with cosine functions. In the second panel (Fig. 6B), the 

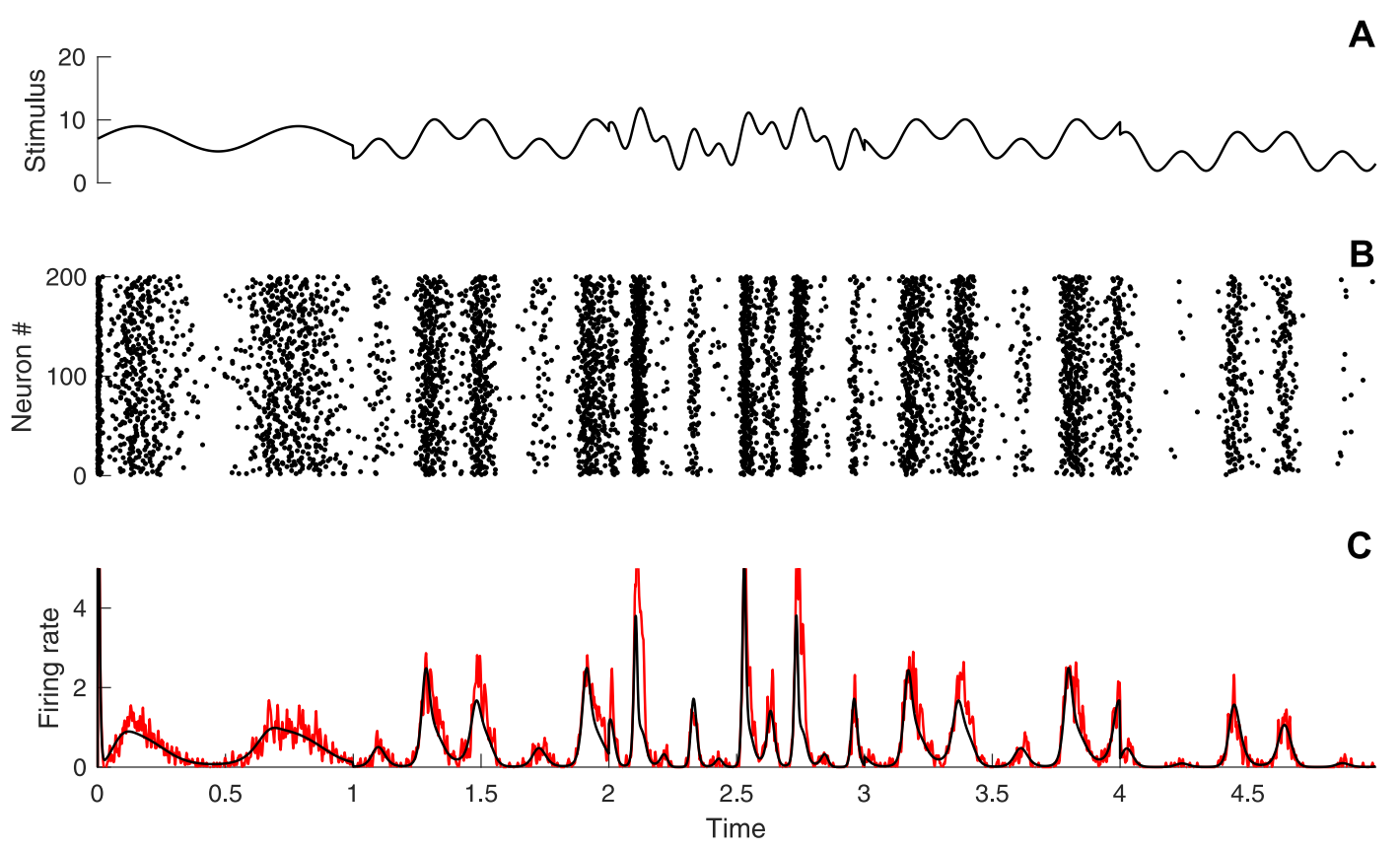

Figure 6 . Comparison between the neural activity generated via simulations of the stochastic process $(2.9),(2.10)$ and the AS system (2.11)-(2.13) for a time dependent stimulus. (A) Time evolution of the stimulus. (B) Activity obtained from several realizations of the stochastic process (2.9). (C) Comparison between the firing rate obtained from the stochastic process (2.9), (2.10) in red, and the one given by the AS system (2.11)-(2.13) in black. A Gaussian was taken as initial condition; the functions and the parameters used in the simulation are: $S(t, a)=\exp (h(t)-V(a))$, with $V(a)=-\log (1-\exp (-a / \tau))$ and $\tau=30$. Here $h(t)$ represents the stimulus that is shown in the first panel.

spiking activity of the cell is shown, from which the firing rate is extracted. A comparison between the firing rate directly computed and the one extracted from the AS system is presented in the last panel (Fig. 6C).

\section{An age and potential structured model. Connections with FP AND AS SYSTEMS}

\subsection{A two-dimensional stochastic model}

The model that we shall introduce here simply takes into account the simultaneous time evolution of both variables: potential and age. In the subthreshold regime, both variables evolve in time independently by each other: the evolution of the potential is described by the NLIF model (2.1), while the age grows linearly with time (2.9). The connection between both variables is obtained at the firing time, which is considered to take place when the potential variable reaches a fixed threshold value. At this moment, the potential is reset to another fix value and the age is reset to zero. We deal therefore with the following two-dimensional stochastic process, where a noise term acts only over the dynamics of the potential variable:

$$
\left\{\begin{array}{l}
\frac{\mathrm{d}}{\mathrm{d} t} v(t)=(\mu(t)-v(t))+\sigma \xi(t), \\
\frac{\mathrm{d}}{\mathrm{d} t} a(t)=1
\end{array}\right.
$$


but affects also the age of the neuron via the firing mechanism:

$$
\text { if } \quad v \geq 1 \text { then } v \mapsto v_{r} \text { and } \quad a \mapsto 0 .
$$

As in the previous section, $\mu(t)$ is the mean of the external stimulus, $\sigma$ is the noise intensity of the external stimulus, and $\xi$ is again a Gaussian white noise

$$
\langle\xi(t)\rangle=0, \quad\left\langle\xi(t) \xi\left(t^{\prime}\right)\right\rangle=\delta\left(t-t^{\prime}\right) .
$$

The probability density function associated to the system (3.1), (3.2) satisfies therefore a two-dimensional Fokker-Planck equation (see [24]):

$$
\frac{\partial}{\partial t} \pi(t, a, v)+\overbrace{\frac{\partial}{\partial a} \pi(t, a, v)+\frac{\partial}{\partial v}[(\mu(t)-v) \pi(t, a, v)]}^{\text {Drift }}-\overbrace{\frac{\sigma^{2}}{2} \frac{\partial^{2}}{\partial v^{2}} \pi(t, a, v)}^{\text {Diffusion }}=0 .
$$

Corresponding to the spiking mechanism (3.2), we impose an absorbing boundary condition at the firing threshold:

$$
\pi(t, a, 1)=0,
$$

while a no-flux boundary condition is taken at the lower boundary:

$$
\lim _{v \rightarrow-\infty}(-\mu+v) \pi(t, a, v)+\frac{\sigma^{2}}{2} \frac{\partial}{\partial v} \pi(t, a, v)=0 .
$$

As a consequence of the diffusion process, the following relation takes place:

$$
\lim _{a \rightarrow+\infty} \pi(t, a, v)=0 .
$$

The flux at the firing threshold

$$
\rho(t, a)=-\frac{\sigma^{2}}{2} \frac{\partial}{\partial v} \pi(t, a, 1)
$$

gives the probability flux of firing at age $a$, and, since once firing occurs, both membrane potential and the age of a neuron are reset in the value $\left(0, v_{r}\right)$, the following boundary condition corresponding to age $a=0$ arises:

$$
\pi(t, 0, v)=\delta\left(v-v_{r}\right) r(t)
$$

where the firing rate of the system is defined by:

$$
r(t)=\int_{0}^{\infty} \rho(t, a) \mathrm{d} a .
$$

Finally, an initial distribution is assumed as known:

$$
\pi(0, a, v)=\pi_{0}(a, v) .
$$



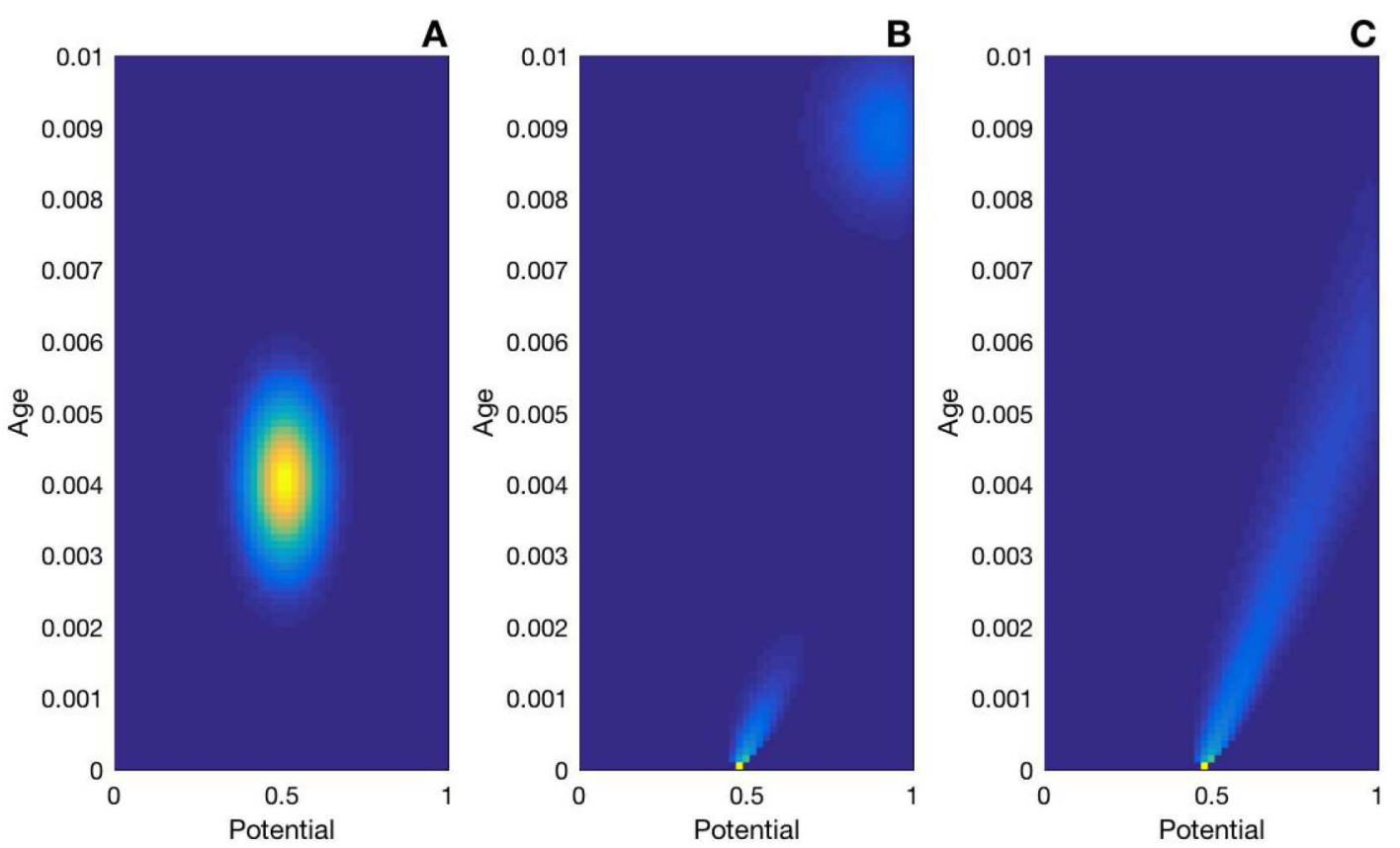

Figure 7. Simulation of the age-potential system (3.3)-(3.6). A two dimensional Gaussian (age-potential) was taken as initial condition; the parameters of the simulation are: $v_{r}=0.5$, $\mu=85, \sigma=1$. The plots show the evolution in time of the solution, respectively at $t=0$, $t=0.5$ and $t=7$ for the respective panels $\mathrm{A}-\mathrm{C}$.

The model is now complete, and one can check directly by integration over the state space that, if the initial distribution satisfies

$$
\int_{-\infty}^{1} \int_{0}^{\infty} \pi_{0}(a, v) \mathrm{d} a \mathrm{~d} v=1
$$

then, at any $t>0$ :

$$
\int_{-\infty}^{1} \int_{0}^{\infty} \pi(t, a, v) \mathrm{d} a \mathrm{~d} v=1
$$

In Figure 7, a simulation of the age-potential system (3.3)-(3.6) is presented. The three panels (A-C) show the evolution in time of the probability density. The simulation starts with a Gaussian as initial condition (the first panel of Fig. 7). Under the influence of the drift term, the density function advances in age, which is clearly seen in the plots of Figure 7. After the spiking process, the age of the neuron is reset to zero and its membrane potential is reset to $v_{r}$. This effect is well perceived in the second panel of Figure 7 .

\subsection{The first passage time problem}

The associated first passage time problem for the probability density of a neuron to be at time $t$ in the state $(a, v)$, given that at the firing time $t-a$ has been in the state $\left(0, v_{r}\right)$, denoted here by $\varphi(t, a, v)$, is therefore 
given by:

$$
\frac{\partial}{\partial t} \varphi(t, a, v)+\frac{\partial}{\partial a} \varphi(t, a, v)+\frac{\partial}{\partial v}[(\mu(t)-v) \varphi(t, a, v)]-\frac{\sigma^{2}}{2} \frac{\partial^{2}}{\partial v^{2}} \varphi(t, a, v)=0
$$

with a pulse boundary condition at the firing time, i.e. at age $a=0$ :

$$
\varphi(t, 0, v)=\delta\left(v-v_{r}\right)
$$

and completed with the boundary conditions:

$$
\begin{gathered}
\varphi(t, a, 1)=0 \\
\lim _{v \rightarrow-\infty}(-\mu(t)+v) \varphi(t, a, v)+\frac{\sigma^{2}}{2} \frac{\partial}{\partial v} \varphi(t, a, v)=0 .
\end{gathered}
$$

As initial condition we impose

$$
\varphi(0, a, v)=\varphi_{0}(a, v)
$$

by assuming that for past times, $t<0$, the external stimulus was constant $\mu(t) \equiv \mu_{0}$; in this case, $\varphi_{0}$ can be taken as the asymptotic value of the solution to (3.7) when $\mu$ is constant.

The corresponding survivor function, i.e. the probability that a neuron did not fire yet at time $t$ and age $a$, is then by definition:

$$
P(t, a)=\int_{-\infty}^{1} \varphi(t, a, v) \mathrm{d} v
$$

and the corresponding inter-spike intervals density (ISI) function is given by the flux at the spiking threshold:

$$
I S I(t, a)=-\frac{\sigma^{2}}{2} \frac{\partial}{\partial v} \varphi(t, a, 1)
$$

It checks out immediately that

$$
P(t, 0)=1
$$

which is natural given the interpretation of the survivor function.

The following relation between the survivor function and the ISI function takes place:

$$
I S I(t, a)=-\frac{D}{D t} P(t, a)=-\left(\frac{\partial}{\partial t} P(t, a)+\frac{\partial}{\partial a} P(t, a)\right) .
$$

In the rest of the paper we shall consider the hazard rate defined as

$$
S(t, a)=\frac{I S I(t, a)}{P(t, a)} .
$$




\subsection{The dual problem}

We shall denote now by $\psi(t, a, v)$ the adjoint function to $\varphi(t, a, v)$, i.e., the solution to the following equation

$$
\frac{\partial}{\partial t} \psi(t, a, v)+\frac{\partial}{\partial a} \psi(t, a, v)+(\mu(t)-v) \frac{\partial}{\partial v} \psi(t, a, v)+\frac{\sigma^{2}}{2} \frac{\partial^{2}}{\partial v^{2}} \psi(t, a, v)=0
$$

with the threshold boundary condition

$$
\psi\left(t, a, v_{r}\right)=\psi(t, a, 1)
$$

completed with

$$
\psi\left(t, 0, v_{r}\right)=\int_{0}^{\infty} I S I(t, a) \psi(t, a, 1) \mathrm{d} a .
$$

Combining the last two conditions, we can consider a more general nonlocal one for $a=0$ :

$$
\psi(t, 0, v)=\int_{0}^{\infty} I S I(t, a) \psi(t, a, v) \mathrm{d} a .
$$

It is straightforward to check that $\psi$ satisfies:

$$
\frac{\partial}{\partial t} \int_{0}^{\infty} \int_{-\infty}^{1} \varphi(t, a, v) \psi(t, a, v) \mathrm{d} v \mathrm{~d} a=0
$$

As usual, the dual problem defined by (3.14)-(3.17) is inverse in time.

\subsubsection{Theoretical connections}

Having set the necessary theoretical framework, we are ready now to introduce our results. The first one introduces a special solution to (3.3)-(3.6), and we shall prove that both models reminded in the previous two sections, i.e. FP model (2.3)-(2.6), respectively the AS model (2.11)-(2.13), although known as different formalizations of noise action over the neural dynamics, can, in fact, be viewed as generated by it. In fact, this solution allows us to see the solution of the FP model (2.3)-(2.6) as an integral transform of the solution to the AS system with a kernel that depends of the solution to the first passage time problem. We need to underline though that the AS system generated by it has a special age-dependent death rate, as it can be seen below. In reverse, our second result allows an inverse transform from the solution to the FP system to the solution to the AS system with the help of the solution to the dual system.

Theorem 3.1. Let $\pi$ be a solution to the system (3.3)-(3.6).

1. The function defined by

$$
\pi(t, a, v)=\frac{\varphi(t, a, v)}{P(t, a)} n(t, a)
$$

is a solution to the system (3.3)-(3.6), where $n(t, a)$ is a solution to (2.11)-(2.13).

2. The probability density function defined as

$$
p(t, v)=\int_{0}^{\infty} \pi(t, a, v) \mathrm{d} a,
$$


is the solution to the FP system (2.3)-(2.6) with the initial repartition given by

$$
p_{0}(v)=\int_{0}^{\infty} \pi_{0}(a, v) \mathrm{d} a .
$$

Let us remind first that we assume the boundedness of the stimulus, i.e. $\alpha \leq \mu(t) \leq \beta$, for all $t \geq 0$, with $\alpha, \beta$ real constants. We shall introduce first the notion of weak solutions to system (2.3)-(2.6) in the sense introduced in [10]. Namely:

Definition 3.2. A pair of nonnegative functions $(p, r)$ such that $p \in L_{+}^{2}((0, T) \times(-\infty, 1)), r \in L_{+}^{2}(0, T)$ is a weak solution to (2.3)-(2.6) if, for any test functions $\xi(t, v) \in L^{2}((0, T) \times(-\infty, 1])$ such that $\frac{\partial^{2}}{\partial v^{2}} \xi(t, v), \frac{\partial}{\partial t} \xi(t, v)$, $(\mu(t)-v) \frac{\partial}{\partial v} \xi(t, v) \in L^{2}((0, T) \times(-\infty, 1)), \xi(T, v)=0$, the following relation takes place:

$$
\begin{aligned}
& \int_{0}^{T} \int_{-\infty}^{1} p(t, v)\left[-\frac{\partial \xi(t, v)}{\partial t}-(\mu(t)-v) \frac{\partial \xi(t, v)}{\partial v}-\frac{\sigma^{2}}{2} \frac{\partial^{2} \xi(t, v)}{\partial v^{2}}\right] \mathrm{d} v \mathrm{~d} t \\
& =\int_{0}^{T} r(t)\left[\xi\left(t, v_{r}\right)-\xi(t, 1)\right] \mathrm{d} t+\int_{-\infty}^{1} p_{0}(v) \xi(0, v) \mathrm{d} v .
\end{aligned}
$$

It is also possible to define the time derivative as follows, by choosing $\Phi(v) \in L^{2}(-\infty, 1)$ such that $\Phi^{\prime}(v), v \Phi^{\prime}(v), \Phi^{\prime \prime}(v) \in L^{2}(-\infty, 1)$ :

$$
\frac{\partial}{\partial t} \int_{-\infty}^{1} p(t, v) \Phi(v) \mathrm{d} v=\int_{-\infty}^{1} p(t, v)\left[(\mu(t)-v) \Phi^{\prime}(v)+\frac{\sigma^{2}}{2} \Phi^{\prime \prime}(v)\right] \mathrm{d} v+r(t)\left[\Phi\left(v_{r}\right)-\Phi(1)\right] .
$$

In the same manner, we can introduce the weak solution for the systems (3.3)-(3.6):

Definition 3.3. A pair of nonnegative functions $(\pi, \rho)$ such that $\pi \in L_{+}^{2}\left((0, T) \times(0, A), L_{+}^{2}(-\infty, 1)\right), \rho \in$ $L_{+}^{2}((0, T) \times(0, A))$ is a weak solution to (3.3)-(3.6), if for any test functions $\Phi(v) \in L^{2}(-\infty, 1)$ such that $\Phi^{\prime}(v), v \Phi^{\prime}(v), \Phi^{\prime \prime}(v) \in L^{2}(-\infty, 1)$, the following relation takes place:

$$
\left(\frac{\partial}{\partial t}+\frac{\partial}{\partial a}\right) \int_{-\infty}^{1} \pi(t, a, v) \Phi(v) \mathrm{d} v=\int_{-\infty}^{1} \pi(t, a, v)\left[(\mu(t)-v) \Phi^{\prime}(v)+\frac{\sigma^{2}}{2} \Phi^{\prime \prime}(v)\right] \mathrm{d} v-\rho(t, a) \Phi(1) .
$$

Proof. 1. The proof is straightforward. Let us formally check that equation (3.3) is satisfied:

$$
\begin{aligned}
\frac{\partial}{\partial t}( & \left.\frac{\varphi(t, a, v)}{P(t, a)} n(t, a)\right)+\frac{\partial}{\partial a}\left(\frac{\varphi(t, a, v)}{P(t, a)} n(t, a)\right) \\
& +\frac{\partial}{\partial v}\left[(\mu(t)-v) \frac{\varphi(t, a, v)}{P(t, a)} n(t, a)\right]-\frac{\sigma^{2}}{2} \frac{\partial^{2}}{\partial v^{2}}\left(\frac{\varphi(t, a, v)}{P(t, a)} n(t, a)\right) \\
= & \left(\frac{\partial}{\partial t} \varphi(t, a, v)+\frac{\partial}{\partial a} \varphi(t, a, v)\right) \frac{n(t, a)}{P(t, a)} \\
& +\left(\frac{\partial}{\partial t} n(t, a)+\frac{\partial}{\partial a} n(t, a)\right) \frac{\varphi(t, a, v)}{P(t, a)} \\
& -\frac{\left.\left(\frac{\partial}{\partial t} P(t, a)+\frac{\partial}{\partial a} P(t, a)\right)\right)}{P(t, a)} \frac{\varphi(t, a, v)}{P(t, a)} n(t, a) \\
& +\frac{\partial}{\partial v}[(\mu(t)-v) \varphi(t, a, v)] \frac{n(t, a)}{P(t, a)}-\frac{\sigma^{2}}{2} \frac{\partial^{2}}{\partial v^{2}} \varphi(t, a, v) \frac{n(t, a)}{P(t, a)}
\end{aligned}
$$




$$
\begin{aligned}
= & \frac{n(t, a)}{P(t, a)}\left(\frac{\partial}{\partial t} \varphi(t, a, v)+\frac{\partial}{\partial a} \varphi(t, a, v)+\frac{\partial}{\partial v}[(\mu(t)-v) \varphi(t, a, v)]-\frac{\sigma^{2}}{2} \frac{\partial^{2}}{\partial v^{2}} \varphi(t, a, v)\right) \\
& -\frac{\varphi(t, a, v)}{P(t, a)}\left(\frac{\partial}{\partial t} n(t, a)+\frac{\partial}{\partial a} n(t, a)+S(t, a) n(t, a)\right) \\
= & 0 .
\end{aligned}
$$

The boundary condition at $a=0$ checks out immediately:

$$
\pi(t, 0, v)=\frac{\varphi(t, 0, v)}{P(t, 0)} n(t, 0)=\delta\left(v-v_{r}\right) r(t)
$$

where

$$
\begin{aligned}
r(t) & =\int_{0}^{\infty} S(t, a) n(t, a) \mathrm{d} a=\int_{0}^{\infty} \frac{n(t, a)}{P(t, a)}\left(-\frac{\sigma^{2}}{2} \frac{\partial}{\partial v} \varphi(t, a, 1)\right) \mathrm{d} a \\
& =\int_{0}^{\infty} \rho(t, a) \mathrm{d} a .
\end{aligned}
$$

2. Let us consider the distributional derivative of what we will denote for now $p(t, v):=\int_{0}^{\infty} \pi(t, a, v) \mathrm{d} a$ :

$$
\begin{aligned}
& \frac{\partial}{\partial t} \int_{-\infty}^{1} \Phi(v) p(t, v) \mathrm{d} v=\frac{\partial}{\partial t} \int_{-\infty}^{1} \Phi(v) \int_{0}^{\infty} \pi(t, a, v) \mathrm{d} a \mathrm{~d} v \\
= & \int_{-\infty}^{1} \Phi(v) \int_{0}^{\infty} \frac{\partial}{\partial t} \pi(t, a, v) \mathrm{d} a \mathrm{~d} v \\
= & \int_{-\infty}^{1} \int_{0}^{\infty}\left\{-\frac{\partial}{\partial a} \pi(t, a, v)-\left[\frac{\partial}{\partial v}[(\mu(t)-v) \pi(t, a, v)]+\frac{\sigma^{2}}{2} \frac{\partial^{2}}{\partial v^{2}} \pi(t, a, v)\right] \Phi(v)\right\} \mathrm{d} a \mathrm{~d} v \\
= & \int_{-\infty}^{1} \pi(t, 0, v) \Phi(v) \mathrm{d} v+\int_{0}^{\infty}\left(-\frac{\sigma^{2}}{2} \frac{\partial}{\partial v} \pi(t, a, 1) \Phi(1)\right) \mathrm{d} a \\
& +\int_{-\infty}^{1} \int_{0}^{\infty} \pi(t, a, v)\left[(\mu-v) \Phi^{\prime}(v)+\frac{\sigma^{2}}{2} \Phi^{\prime \prime}(v)\right] \mathrm{d} a \mathrm{~d} v .
\end{aligned}
$$

We remind now that we defined:

$$
\begin{aligned}
& \rho(t, a)=-\frac{\sigma^{2}}{2} \frac{\partial}{\partial v} \pi(t, a, 1), \\
& \pi(t, 0, v)=\delta\left(v-v_{r}\right) r(t), \\
& r(t):=\int_{0}^{\infty} \rho(t, a) \mathrm{d} a .
\end{aligned}
$$

Then, replacing the last formulae in the previous relation, we get:

$$
\begin{aligned}
& \frac{\partial}{\partial t} \int_{-\infty}^{1} \Phi(v)\left(\int_{0}^{\infty} \pi(t, a, v) \mathrm{d} a\right) \mathrm{d} v \\
& =\int_{-\infty}^{1}\left(\int_{0}^{\infty} \pi(t, a, v) \mathrm{d} a\right)\left[(\mu-v) \Phi^{\prime}(v)+\frac{\sigma^{2}}{2} \Phi^{\prime \prime}(v)\right] \mathrm{d} v \\
& +r(t)\left[\Phi\left(v_{r}\right)-\Phi(1)\right],
\end{aligned}
$$


which proves that $p$ defined by (3.19) is solution to (2.3)-(2.6). We end the proof by noticing that taking $\Phi(v) \equiv 1$, the conservation property

$$
\int_{-\infty}^{1} p(t, v) \mathrm{d} v=1
$$

is fulfilled.

Remark 3.4. Note that under the assumptions of Theorem 3.1, the solution to the AS system is similarly obtained as:

$$
n(t, a)=\int_{-\infty}^{1} \pi(t, a, v) \mathrm{d} v
$$

Theorem 3.5. The function defined via the integral

$$
n(t, a)=\int_{-\infty}^{1} \chi(t, a, v) p(t, v) \mathrm{d} v
$$

where

$$
\chi(t, a, v)=P(t, a) \psi(t, a, v),
$$

is a solution to the age-structured system (2.11)-(2.13) as soon as

$$
n_{0}(a)=\int_{-\infty}^{1} \chi_{\infty}(a, v) p_{0}(v) \mathrm{d} v
$$

with $\chi_{\infty}$ solution to the corresponding stationary adjoint system.

Remark 3.6. At this point, there is no evidence that the dual problem for the adjoint function $\psi$ is well posed. However, there is evidence that it is possible to prove the well posedness for the associated problem in $\chi$, under the assumption of finite potential and age intervals using a method given in [17]. This result is in working progress. In the following, therefore, the computations are at the formal level.

Proof. One can check directly that, setting

$$
S(t, a)=\frac{I S I(t, a)}{P(t, a)}
$$

the function defined by (3.24) satisfies the following system:

$$
\frac{\partial}{\partial t} \chi(t, a, v)+\frac{\partial}{\partial a} \chi(t, a, v)+(\mu(t)-v) \frac{\partial}{\partial v} \chi(t, a, v)+\frac{\sigma^{2}}{2} \frac{\partial^{2}}{\partial v^{2}} \chi(t, a, v)+S(t, a) \chi(t, a, v)=0
$$

with

$$
\chi\left(t, a, v_{r}\right)=\chi(t, a, 1) .
$$


Moreover, by imposing the following condition

$$
\int_{0}^{\infty} \chi(t, a, v) \mathrm{d} a=1
$$

the non-local boundary condition

$$
\chi(t, 0, v)=\int_{0}^{\infty} S(t, a) \chi(t, a, v) \mathrm{d} a
$$

follows from equation (3.27) by directly integrating it over the age-interval.

To verify that the function defined by (3.23) is a solution to the AS system, we can formally compute:

$$
\begin{aligned}
& \frac{\partial}{\partial t} \int_{-\infty}^{1} \chi(t, a, v) p(t, v) \mathrm{d} v+\frac{\partial}{\partial a} \int_{-\infty}^{1} \chi(t, a, v) p(t, v) \mathrm{d} v \\
& =\int_{-\infty}^{1}\left\{\left[\frac{\partial}{\partial t} \chi(t, a, v)+\frac{\partial}{\partial a} \chi(t, a, v)\right] p(t, v)+\chi(t, a, v) \frac{\partial}{\partial t} p(t, v)\right\} \mathrm{d} v \\
& =\int_{-\infty}^{1}\left[\frac{\partial}{\partial t} \chi(t, a, v)+\frac{\partial}{\partial a} \chi(t, a, v)\right] p(t, v) \mathrm{d} v \\
& \quad+\underbrace{\int_{-\infty}^{1} \frac{\partial}{\partial v}\left\{[-(\mu(t)-v) p(t, v)]+\frac{\sigma^{2}}{2} \frac{\partial^{2}}{\partial v^{2}} p(t, v)+\delta\left(v-v_{r}\right) r(t)\right\} \chi(t, a, v) \mathrm{d} v}_{I_{2}} .
\end{aligned}
$$

Integrating by parts in $I_{2}$, we get:

$$
I_{2}=\int_{-\infty}^{1}\left[(\mu(t)-v) \frac{\partial}{\partial v} \chi(t, a, v)+\frac{\sigma^{2}}{2} \frac{\partial^{2}}{\partial v^{2}} \chi(t, a, v)\right] p(t, v) \mathrm{d} v-\chi(t, a, 1) r(t)+\chi\left(t, a, v_{r}\right) r(t) .
$$

Using now the boundary condition (3.28) and replacing $I_{2}$ in the expression above, it follows that:

$$
\frac{\partial}{\partial t} \int_{-\infty}^{1} \chi(t, a, v) p(t, v) \mathrm{d} v+\frac{\partial}{\partial a} \int_{-\infty}^{1} \chi(t, a, v) p(t, v) \mathrm{d} v=-S(t, a) \int_{-\infty}^{1} \chi(t, a, v) p(t, v) \mathrm{d} v
$$

therefore, the function defined by (3.23) is a solution to (2.11).

The boundary condition checks out immediately:

$$
\begin{aligned}
n(t, 0) & =\int_{-\infty}^{1} \chi(t, 0, v) p(t, v) \mathrm{d} v \\
& =\int_{0}^{\infty} S(t, a) \int_{-\infty}^{1} \chi(t, a, v) p(t, v) \mathrm{d} v \mathrm{~d} a \\
& =\int_{0}^{\infty} S(t, a) n(t, a) \mathrm{d} a .
\end{aligned}
$$


Last, the conservation property is also verified directly since

$$
\begin{aligned}
& \int_{0}^{\infty} n(t, a) \mathrm{d} a=\int_{0}^{\infty} \int_{-\infty}^{1} \chi(t, a, v) p(t, v) \mathrm{d} v \mathrm{~d} a \\
& =\int_{-\infty}^{1} p(t, v) \int_{0}^{\infty} \chi(t, a, v) \mathrm{d} a \mathrm{~d} v=\int_{-\infty}^{1} p(t, v) \mathrm{d} v=1 .
\end{aligned}
$$

\section{Discussion}

The present paper is thought as a continuation of our previous work [19, 20], where we have uncovered a connection between the NLIF and the escape rate model in the context of a time independent stimulus. We proved there that the corresponding solutions to the FP equation and the AS system can be mapped one-toanother via integral transforms. In the present paper, we wish to go further by considering stimuli that are time dependent, in which case our previous results do not apply. We did find out though that it is possible to recover both the NLIF and the escape rate models starting from a more general, two dimensional stochastic model. Our work is thus a first attempt to describe a general theoretical framework that can be used to understand key issues related to the mathematical treatment of neural variability.

The importance of our results is mostly theoretical. Our conclusion is that it is always possible to adjust parameters such that the NLIF and the escape rate models exhibit the same statistics. This finding enables us to give one possible explanation for the reported similarities between the two frameworks [34]. The choice of using any of it would then be influenced only by which of the variables - age or membrane potential - one wants to take into account.

From a practical point of view, finding an explicit formula of the correspondence, or, at least, an approximation would be a plus. This is a difficult task, as we have seen $[19,20]$, such a mapping relies on the first passage time problem for which no explicit solution has been found. Even though in this paper we do not address the issue, a possible biological application would be the connection between the external stimulus $\mu$ which is explicit in the FP equation and the spiking activity of the corresponding AS model. To this aim, let us consider the following hypothetical situation:

Let us consider a population of similar NLIF neurons that is subject to an external input received from another population of neurons. Suppose that the activity of the population is recorded by a network of electrodes that can record individually the firing of some neurons. Assuming that the population considered is large enough, we can have a good statistical representation of the whole population. The recordings provide a number of neurons $n_{\text {obs }}(t, a)$ that had a spike at time $t-a$ and none since then. The issue addressed then is the following: Given $n_{\text {obs }}(t, a)$ and knowing that for $t<0, \mu(t) \equiv \mu_{0}$, can we estimate the stimulus $\mu(t)$ acting over the population? We think that our transform (3.23) can be used in such a situation. This can be done by defining in a classical manner the quadratic error function:

$$
J(\mu)=\int_{0}^{T} \int_{0}^{\infty}\left\|n_{o b s}(t, a)-n(t, a ; \mu)\right\|^{2} \mathrm{~d} a \mathrm{~d} t
$$

which is to be minimized with respect to $\mu$. Here $n(t, a ; \mu)$ is defined via the integral transform (3.23), relation that uses the solutions $\psi(t, a, v)$ and $p(t, v)$ that satisfy systems having explicit dependence on the external stimulus. The issue presented here is currently under our investigations.

Although the FP equation in neuronal dynamics context has been studied over the past few years and qualitative results regarding its solutions have been proven $[10,13,14,16,22]$, the AS systems theory has received a lot of attention in the last decades, see monographs $[27,37]$ for example, and different associated 
control problems have been considered [2]. There is, therefore, a big advantage from a mathematical point of view to privilege its use in neuronal modelling.

Let us finally conclude by saying that our work opens several pathways for future research. From our side, the most exciting one is the investigation of emergent properties of neural networks. Both formalisms (FP and AS) have been employed to describe neural circuits in the mean-field approximation (see $[4,5,10,11,22,35]$ for the FP and see $[12,15,21,30-33]$ for the AS), and both of them have generated key insights in neuroscience, especially in the quest of the underlying mechanism of neural synchronization $[18,33]$. It would be therefore relevant to use our new framework to investigate the links and differences between the two approaches, especially when conclusions are contradictory.

\section{REFERENCES}

[1] L.F. Abbott, Lapique's introduction of the integrate-and-fire model neuron (1907). Brain Res. Bull. 50 (1999) $303-304$.

[2] S. Aniţa, Analysis and Control of Age-Dependent Population Dynamics. Kluwer Academic Publishers, Dordrecht (2000).

[3] N. Brunel and M.C. van Rossum, Lapicque's 1907 paper: from frogs to integrate-and-fire. Biol. Cybern. 97 (2007) $341-349$.

[4] N. Brunel, Dynamics of sparsely connected networks of excitatory and inhibitory spiking neurons. J. Comput. Neurosci. 8 (2000) 183-208.

[5] N. Brunel and V. Hakim, Fast global oscillations in networks of integrate-and-fire neurons with low firing rates. Neural Comput. 11 (1999) 1621-1671.

[6] N. Brunel and V. Hakim, Fokker-planck equation. Encyclopedia of Computational Neuroscience. Springer, New York (2015), 1222-1226, 2015.

[7] N. Brunel and M.C.W. van Rossum, Quantitative investigations of electrical nerve excitation treated as polarization. Biol. Cybern. 97 (2007) 341-349.

[8] A.N. Burkitt. A review of the integrate-and-fire neuron model: I. homogeneous synaptic input. Biological Cybernetics, 95 (2006) 1-19, .

[9] A.N. Burkitt, A review of the integrate-and-fire neuron model: II. inhomogeneous synaptic input and network properties. Biol. Cybern. 95 (2006) 97-112.

[10] M. Cáceres, J.A. Carrillo and B. Perthame, Analysis of nonlinear noisy integrate \& fire neuron models: blow-up and steady states. J. Math. Neurosci. 1 (2011) 7.

[11] M.J. Cáceres and R. Schneider, Analysis and numerical solver for excitatory-inhibitory networks with delay and refractory periods. ESAIM: M2AN 52 (2018) 1733-1761.

[12] J.A. Cañizo and H. Yoldaş, Asymptotic behaviour of neuron population models structured by elapsed-time. Nonlinearity 32 (2019) 464-495.

[13] J.A. Carillo, B. Perthame, D. Salort and D. Smets, Qualitative properties of solutions for the noisy integrate and fire model in computational neuroscience. Nonlinearity 8 (2015) 9.

[14] J.A. Carrillo, M.D.M. Gonzalez, M.P. Gualdani and M.E. Schonbek, Classical solutions for a nonlinear fokker-planck equation arising in computational neuroscience. Commun. Partial Differ. Equ. 38 (2013) 385- 409.

[15] J. Chevalier, M.J. Caceres, M. Doumic and P. Reynaud-Bouret, Microscopic approach of a time elapsed neural model. Math. Models Methods Appl. Sci. 25 (2015) 2669-2719.

[16] F. Delarue, J. Inglis, S. Rubenthaler and E. Tanré, Global solvability of a networked integrate-and-fire model of McKean-Vlasov type. Ann. Appl. Probab. 25 (2015) 2096-2133.

[17] N. Dokuchaev, On recovering parabolic diffusions from their time averages. Preprint arXiv: 1609.01890 (2017).

[18] G Dumont and J Henry, Synchronization of an excitatory integrate-and-fire neural network. Bull. Math. Biol. 75 (2013) $629-48$.

[19] G. Dumont, J. Henry and C.O. Tarniceriu, Noisy threshold in neuronal models: connections with the noisy leaky integrate and - fire model. J. Math. Biol. 73 (2016) $1413-1436$.

[20] G. Dumont, J. Henry and C.O. Tarniceriu, Theoretical connections between neuronal models corresponding to different expressions of noise. J. Theor. Biol. 406 (2016) 31-41.

[21] G. Dumont, A. Payeur and A. Longtin, A stochastic-field description of finite-size spiking neural networks. PLOS Comput. Biol. 13 (2017) 1-34

[22] G. Dumont and P. Gabriel, The mean-field equation of a leaky integrate-and-fire neural network: measure solutions and steady states. Preprint arXiv: 1710.05596 (2020).

[23] A.A. Faisal, L.P. Selen and D. Wolpert, Noise in the nervous system. Nat. Rev. Neurosci. 9 (2008) 292-303.

[24] C.W. Gardiner, Handbook of Stochastic Methods for Physics, Chemistry and Natural Sciences. Springer, Berlin (1996)

[25] W. Gerstner and J.L. Van Hemmen, Associative memory in a network of 'spiking' neurons. Network 3 (1992) $139-164$.

[26] W. Gerstner and W. Kistler, Spiking neuron models. Cambridge University Press, Cambridge (2002).

[27] M. Iannelli, Mathematical Theory of Age-Structured Population Dynamics. CNR Applied Mathematics Monographs, Vol. 7. Giardini editori e stampatori, Pisa (1995).

[28] P. Langevin, Sur la théorie du mouvement brownien. C. R. Acad. Sci. (Paris) 146 (1908) 530-533.

[29] A. Longtin, Neuronal noise. Scholarpedia 8 (2013) 1618. 
[30] C. Meyer and C.A. van Vreeswijk, Temporal correlations in stochastic networks of spiking neurons. Neural Comput. 10 (2002) $1321-1372$.

[31] S. Mischler and C. Quiñinao, Weak and strong connectivity regimes for a general time elapsed neuron network model. J. Stat. Phys. 173 (2018) 77-98.

[32] K. Pakdaman, B. Perthame and D. Salort, Dynamics of a structured neuron population. Nonlinearity 23 (2009) $23-55$.

[33] K. Pakdaman, B. Perthame and D. Salort, Relaxation and self-sustained oscillations in the time elapsed neuron network model. SIAM J. Appl. Math. 73 (2013) 1260-1279.

[34] H.E. Plesser and W. Gerstner, Noise in integrate-and-fire neurons: from stochastic input to escape rates. Neural Comput. 12 (2000) 367-384.

[35] A. Renart, N. Brunel and X.-J Wang, Mean-Field Theory of Irregularly Spking Neuronal Populations and Working Memory in Recurrent Cortical Networks, Chapter 15 in Computational Neuroscience: A comprehensive Approach, Mathematical Biology and Medicine Series. Chapmann\&Hall/CRC, Boca Raton (2004).

[36] L.S. Tsimring, Noise in biology. Rep. Prog. Phys. 77 (2014) 026601.

[37] G. Webb, Theory of Nonlinear Age-Dependent Population Dynamics. Marcel Dekker, New York (1985). 